\title{
La pedagogía social, una mirada para la resolución de un conflicto socio-ambiental ${ }^{1}$
}

\author{
Claudia Amparo Torres Ángel ${ }^{2}$ \\ Universidad de la Sabana - Bogotá, Colombia \\ claudiaamparo@gmail.com \\ María Consuelo Torres Ángel ${ }^{3}$ \\ Universidad de la Sabana - Bogotá, Colombia \\ mtorresangel@yahoo.com
}

1 Artículo derivado de la investigación "Lecciones aprendidas para la pedagogía social: sistematización de un proyecto socioambiental", producto final de la maestría en pedagogía de la Universidad de la Sabana, octubre de 2013.

2 Enfermera comunitaria de la Universidad Nacional. Especialista en desarrollo social y participación comunitaria de la Universidad del Tolima . Aspirante al título de maestría en Pedagogía de la Universidad de la Sabana, 2013.

3 Licenciada en educación preescolar de la Universidad Los Libertadores. Aspirante al título de maestría en Pedagogía de la Universidad de la Sabana, 2013. 


\title{
La pedagogía social, una mirada para la resolución de un conflicto socio-ambiental
}

\section{Resumen}

Para este fin, se utilizaron como instrumentos de recolección de datos, encuestas, entrevistas y talleres que permitieron un acercamiento a los actores involucrados en el proceso y la obtención de la información requerida para documentar el caso. El estudio permitió hacer evidentes los aprendizajes adquiridos a lo largo del proceso, por parte de los participantes de la comunidad y de la empresa, y las enseñanzas sobre la educación ambiental y la participación comunitaria, factores fundamentales en la intervención del conflicto y en la resolución de un conflicto socio-ambiental. De esta forma, se logró la recuperación de este ecosistema, y se puso en escena el valor incalculable de la pedagogía social para el mejoramiento de la producción, dentro del marco del desarrollo sostenible.

De igual manera, se identificó que la propuesta formativa desarrollada por las empresas, se concretó en procesos autónomos de construcción de comunidad, que permiten hablar de una pedagogía que se construye socialmente a partir del diálogo de saberes y del reconocimiento de las prácticas y sus posibilidades de transformación.

Palabras clave: pedagogía social, educación ambiental, participación comunitaria, conflicto socioambiental, sistematización.

\section{Social pedagogy, perspective for a socio-environmental conflict resolution}

\begin{abstract}
This article describes the experiences of socio-environmental conflict resolution between the companies involved in the management of reservoir Tominé and the peasants from the surrounding villages, who used to graze their cattle on the banks of it, contributing to its deterioration and getting poor results in the livestock production. Since this is a valuable experience, it is considered interesting to systematize the way conflict resolution in both the experience from social pedagogy, carrying out a research within the framework a case study, qualitative method assumed as the most appropriate for studying the ecological, social and economic impact emanating from the results of this process. It also highlights the diversity of interests of the players who managed to arrange common alternative solutions.
\end{abstract}

For this purpose were used as data collection instruments, surveys, interviews and workshops that allowed an approach to the actors involved in the process and obtain the information required to support the case.

The study allowed us to demonstrate the knowledge acquired from throughout the whole process by the community and company participants and the teachings on environmental education and community participation, key factors in conflict intervention and resolution of conflict social-environmental conflict. Therefore, it was possible to recover an ecosystem, thereby staging the incalculable value of social pedagogy to ameliorate production within the confines of a sustainable development.

Similarly, it was found that the training approach developed by the companies, took shape in autonomous processes of community building that paves the way to talk of a pedagogy that is socially constructed from the dialogue of knowledge and recognition of the practices and their ability to transform.

Keywords: Social pedagogy, environmental education, community participation, socio-environmental conflict, systematization

\section{A pedagogia social, uma olhada para a resolução de um conflito socioambiental}

\section{Resumo}

Este artigo coleta a experiência da resolução do conflito socioambiental entre as empresas responsáveis da administração da barragem de Tominé e os agricultores dos povoados próximos, que pastoreavam seu gado no banco dela, causando a deterioração ambiental e gerando resultados baixos na produção pecuária. Vendo que essa é uma experiência importante, achou-se interessante sistematizar a maneira de resolução do conflito no campo da experiência da pedagogia social, ao realizar uma pesquisa orientada pelos parâmetros do estudo de caso e a metodologia qualitativa, pois essa última é a metodologia ideal para conhecer os impactos ecológicos, sociais e econômicos, e para destacar os diferentes interesses dos atores que puderam organizar alternativas de solução.

Para esse fim, foram usadas pesquisas, ferramentas de coleta de dados, entrevistas e atividades que permitiram uma aproximação aos atores envolvidos no processo e na obtenção da informação precisada para documentar o caso. $O$ estudo permitiu expor as aprendizagens dos participantes da comunidade e da empresa obtidas durante todo o processo, e os ensinamentos sobre a educação ambiental e a participação na comunidade, elementos fundamentais na intervenção do conflito e na resolução de um conflito socioambiental. Por isso, foi possível a recuperação de um ecossistema, e foi exibido o valor incalculável da pedagogia social para a melhora da produção, dentro do âmbito do desenvolvimento sustentável.

Do mesmo modo, identificou-se a proposta formativa desenvolvida pelas empresas, especificou-se nos processos autônomos de construção de comunidade, que permitem falar de uma pedagogia construída socialmente a partir do diálogo de saberes e do reconhecimento das práticas e suas possibilidades de transformação.

Palavras chave: Pedagogia social, educação ambiental, participação comunitária, conflito socioambiental, sistematização. 


\section{Introducción}

Según lo ha determinado la convención Ramsar ${ }^{4}$, los embalses son considerados humedales artificiales y por tanto ecosistemas muy productivos, por la riqueza de materia orgánica que almacenan y porque favorecen el desarrollo de gran diversidad de fauna y flora. Sin embargo, se encuentran en peligro de extinción y en Colombia una de las situaciones que más los afecta, es el desarrollo de actividades agropecuarias en sus alrededores (Eccosocial, 2012). Específicamente en Tominé, las comunidades campesinas estaban utilizando el embalse y sus alrededores como zona de pastoreo y abrevadero del ganado, generando un impacto negativo sobre el ecosistema y obteniendo bajo rendimiento en la producción pecuaria. Buscando disminuir los impactos sobre el embalse, y mejorar las condiciones productivas de las comunidades campesinas aledañas al embalse, las empresas encargadas de su protección y manejo, desarrollaron un proceso de resolución del conflicto de manera participativa con dichas comunidades. La investigación adelantada, partió del interés en conocer los aportes pedagógicos que hace una experiencia de educación ambiental y participación comunitaria, al resolver un conflicto socio-ambiental vinculado a la recuperación del ecosistema y al mejoramiento de la producción en el marco del desarrollo sostenible.

\section{Conceptos básicos que orientaron el trabajo}

\section{Pedagogía social}

En este estudio, la pedagogía social se planteó como el conjunto de proposiciones teóricas y metodológicas, enfoques, estrategias y técnicas, para comprender el proceso educativo e incidir efectivamente sobre él (García, 2012). Así mismo, se basó en planteamientos importantes como comprender las interacciones entre el individuo y su medio como "espacio vital" (Fermoso, 1994) y entenderla como acción pedagógica intencional y reglamentada, definida por la realidad social y el contexto, lo cual hace referencia a la necesidad de concebir al hombre en el marco de su comunidad, explorando y actuando en conjunto para obtener resultados en pro de su calidad de vida (Petrus, 1997).

Para el caso que nos ocupa, la pedagogía social resultó pertinente para promover una relación de la comunidad con su medio de manera más cuidadosa si afectar su supervivencia, al tiempo que movilizó a los actores empresariales en torno al conocimiento de la comunidad, sus necesidades y la búsqueda de acciones

4 La Convención Ramsar, es un tratado intergubernamental que sirve de marco para la acción nacional y la cooperación internacional en pro de la conservación y el uso racional de los humedales y sus recursos. 
favorecedoras del medio y de los distintos actores.

\section{Educación y Educación Ambiental}

En cuanto al concepto de educación se partió del planteamiento de Freire quien dice que "[...] la acción educativa es una acción transformadora [...]" que implica praxis, reflexión y acción del hombre sobre el mundo para transformarlo (Freire 1980, en Vega, 2011). De igual manera se tuvo en cuenta que la educación, y muy particularmente la educación ambiental, juega un papel fundamental en el proceso de construcción de un nuevo futuro (Pujol, nd), y que por tanto, ésta se considera como estrategia pedagógica privilegiada para el abordaje de los conflictos socioambientales. Otro aporte fundamental, que además le da fuerza a los resultados obtenidos, es el planteamiento de Lacki (2012), quien dice que la educación y la organización social son la base fundamental para que los campesinos salgan adelante con sus actividades productivas, sin depender de los créditos, subsidios y políticas globales.

En esta medida, la educación ambiental posibilita contar con un conocimiento amplio sobre la necesidad de equilibrio del hombre con el medio ambiente, para que su explotaciçon no lo dañe y para que el hombre pueda obtener sus beneficios, preservándolo.

\section{Desarrollo sostenible y sustentabilidad}

El desarrollo sostenible es aquel que posibilita el crecimiento económico, la elevación de la calidad de vida y el bienestar social, sin agotar la base de los recursos en que se sustenta, ni deteriorar el medio ambiente, para que las futuras generaciones puedan también utilizarlos para la satisfacción de sus propias necesidades. Supone la sustentabilidad (soporte para el sustento) en términos ecológicos, sociales, económicos y geográficos o territoriales. En el proyecto, este concepto resulta fundamental para que ser logre el equilibrio mencionado, y la relación del hombre con su medio pueda establecerse en forma armónica, beneficiándose mutuamente. Es un aprendizaje para toda la vida, al permitir a los actores relaciones mutuas, haciendo converger sus intereses y atender sus diversas necesidades, sin afectar el medio de manera nociva.

\section{Conflicto socio-ambiental}

Aunque la resolución de conflictos puede tener acepciones distintas para cada persona según el oficio, momento o interés. Para nuestros propósitos, resolución de conflicto significa la puesta en escena de intereses diversos y el encuentro de alternativas de trabajo compatibles, que llevaron a una puesta en común, con ayuda de métodos analíticos que se dirigen a la raíz del problema y se orienta a un resultado que de acuerdo con las partes implicadas, es una solución permanente del problema 
(Burton, n.d.). En el caso que ocupa a este estudio, el conflicto socio-ambiental se identificó en el choque de intereses entre las empresas encargadas del manejo del embalse y la producción de energía y los campesinos de la zona aledaña al embalse, por el uso de éste y sus alrededores.

\section{Descripción de la metodología utilizada para la investigación}

la investigación seenmarcó en las metodologías cualitativas, centrándoseespecíficamente en el estudio de caso entendido como el estudio de la particularidad y de la complejidad de un caso singular, para llegar a comprender su actividad en circunstancias concretas (Stake, en Del Castillo et al, 1998). De acuerdo con este enfoque, para la obtención de información se propuso la realización de encuestas, entrevistas y talleres en donde además del acercamiento con la comunidad, se desarrollaron ejercicios como la reconstrucción de la línea del tiempo y la cartografía social.

Para la selección de la muestra se planteó el trabajo con diferentes grupos de edad, género y rol dentro de la comunidad y en el desarrollo del proceso, para tener distintos puntos de vista de la experiencia vivida. En las entrevistas se incluyeron representantes de las empresas vinculadas al proceso, quienes aportaron también su punto de vista acerca del mismo.

Para el estudio se realizaron 11 entrevistas distribuidas así:

- 5 a representantes de Asosantuario

- 2 a representantes de Cooptominé

- 4 a representantes de las empresas vinculadas al proceso

Se realizaron 42 encuestas, 23 a representantes de Asosantuario y 19 a Cooptominé. En cuanto a los talleres, fueron tres en total:

- 1 de reconstrucción de la línea del tiempo con la participación de 28 personas de las dos organizaciones.

- 1 de cartografía social con 25 personas representantes de Asosantuario.

- 1 de cartografía social con 28 representantes de Cooptominé.

\section{Categorías de análisis}

Como categorías de análisis que contribuyeron a la indagación y fueron el contenido de los instrumentos mencionados, se propusieron las que se presentan en la tabla 1 , con su respectiva síntesis: 


\begin{tabular}{|c|c|c|}
\hline Categorías & Subcategorías & Síntesis \\
\hline $\begin{array}{l}\text { Saber pedagógico } \\
\text { comunitario }\end{array}$ & Educación & $\begin{array}{l}\text { Relación constante entre teoría y práctica, en } \\
\text { donde las reflexiones fundamentan la práctica y } \\
\text { ésta a su vez, aporta elementos para la reflexión } \\
\text { teórica. }\end{array}$ \\
\hline \multirow{3}{*}{$\begin{array}{l}\text { Educación } \\
\text { ambiental }\end{array}$} & $\begin{array}{l}\text { Individuo - } \\
\text { ambiente }\end{array}$ & $\begin{array}{l}\text { Relación indisoluble hombre-medio. } \\
\text { Si se entiende el ambiente no sólo como el } \\
\text { espacio en }\end{array}$ \\
\hline & $\begin{array}{l}\text { Comunidad - } \\
\text { ambiente }\end{array}$ & $\begin{array}{l}\text { que se desarrolla la vida, sino como la interrela- } \\
\text { ción entre los aspectos biofísicos, sociales y cul- } \\
\text { turales, es claro que las personas y por ende las } \\
\text { comunidades hacen parte de esa misma red de } \\
\text { relaciones interdependientes e indisolubles y de } \\
\text { sus acciones, depende la conservación del equi- } \\
\text { librio ambiental. }\end{array}$ \\
\hline & $\begin{array}{l}\text { Buenas prácti- } \\
\text { cas medioam- } \\
\text { bientales }\end{array}$ & $\begin{array}{l}\text { Prácticas que posibilitan el equilibrio entre la } \\
\text { comunidad y el ambiente. En general todas las } \\
\text { acciones que resolviendo las necesidades básicas } \\
\text { humanas, minimicen los impactos negativos so- } \\
\text { bre el entorno. }\end{array}$ \\
\hline $\begin{array}{l}\text { Construcción de co- } \\
\text { munidad }\end{array}$ & $\begin{array}{l}\text { Auto percep- } \\
\text { ción como } \\
\text { comunidad }\end{array}$ & $\begin{array}{l}\text { Proceso que busca la unión para la solución de } \\
\text { los problemas comunes, desarrollando lideraz- } \\
\text { go, haciendo alianzas, nutriendo redes sociales y } \\
\text { generando confianza entre los miembros para el } \\
\text { trabajo conjunto y el logro de los objetivos pro- } \\
\text { puestos. }\end{array}$ \\
\hline
\end{tabular}

\section{Resultados}

En primer lugar, cabe destacar la satisfacción y orgullo con que la gran mayoría de las personas que participaron en este estudio, hablan sobre su organización. Asosantuario y Cooptominé hoy son valoradas y queridas por sus asociados, pues han traído a sus vidas mayores ingresos y mejores condiciones para su actividad productiva.

A pesar de los pequeños inconvenientes que han tenido, por lo que algunos consideran desaciertos, en términos generales los ingresos han mejorado entre $30 \%$ y $50 \%$, lo cual es bastante significativo. Esto sin contar, los otros valores agregados que obtuvieron en cada ejercicio, como el aprendizaje que les ha quedado respecto a las buenas prácticas de ordeño, el mejoramiento genético de sus reses, el manejo de las praderas y los procesos para el manejo de la alimentación del ganado, así 
como el impulso y los beneficios de trabajar en conjunto con la comunidad y sentir el respaldo de una organización. El estar unidos y organizados, también les ha abierto puertas para acceder a recursos importantes por parte de las entidades del Estado, lo cual les ha dado grandes impulsos en momentos importantes. Sin embargo, algunos consideran que es necesario mayor apoyo por parte de estas entidades, y que el estar organizados debería traer mayores beneficios, no solo a la organización, sino también a nivel personal.

Tener una estabilidad en cuanto a quien les compra le leche directamente y evitar así los intermediarios, les da mayor tranquilidad y ganancia, les permite organizar mejor su trabajo y estar seguros de que la leche se venderá. Sin embargo, esperan que las empresas compradores valoren más su trabajo y les paguen a mejor precio la leche. Otro punto interesante, es el manejo del liderazgo, sobre todo en Asosantuario, ya que quienes están a la cabeza de las organizaciones han sabido orientar adecuadamente el manejo de las mismas, buscando siempre el consenso y las mejores oportunidades para todos. Así mismo, los asociados hoy reconocen el buen desempeño de sus representantes y por lo tanto, los siguen reeligiendo para seguir al frente de la organización.

En términos generales, valoran el apoyo recibido por la empresa operadora contratada por las empresas: Fundesot (fundación para el desarrollo sostenible), tanto por las capacitaciones recibidas, como por la orientación para la conformación de las organizaciones, y reconocen el proceso educativo, como la base fundamental para estar en el lugar que están hoy. La mayoría considera que la relación con la Empresa ha cambiado y mejorado. Únicamente en Cooptominé, hay descontento con la manera en que la Empresa trata a las personas de la comunidad, se sienten algunas veces irrespetados por quienes les exigen papeles para acceder a los predios del embalse a cortar el pasto para el ganado y consideran que esta empresa debería asumir su responsabilidad social con acciones que respondan a otras necesidades de la comunidad.

También algunos, especialmente de Cooptomine, piensan que el hecho de sacar el ganado de los terrenos aledaños al embalse no ha disminuido el buchón de acuerdo con lo esperado y por el contrario sufren con el deterioro de las praderas, el ruido constante de la máquina que corta el buchón y el mal olor que generan estos residuos en las orillas del embalse. Algunos dicen, que lo único bueno de todo este proceso ha sido su organización. Asosantuario por el contrario, valora los aportes recibidos a través de la empresa y reconoce estos logros como fruto del proceso promovido por las empresas vinculadas al embalse, y básicamente por el trabajo realizado por Fundesot. Es evidente, que la relación con las Empresas ha mejorado desde la organización de las asociaciones, pues hay respaldo, respeto y reconocimiento con su asociación. 
Según los resultados de los talleres de cartografía social, Asosantuario plantea los cambios como mejoría del entorno, del embalse y de las condiciones de vida de las personas de la comunidad, mientras que Cooptominé considera que antes de este proceso, las cosas para la gente y en el entorno estaban mejor. De todas maneras cada uno se siente orgulloso de su organización y se sienten bien de haber elegido el tipo de organización que conformaron (asociación y cooperativa), pues consideran les ofrece las condiciones que mejor se ajustan a sus intereses. También es importante, el reconocimiento que hay acerca del trabajo colectivo, la unión y las alianzas para obtener mejores resultados para todos. Es también interesante ver que los logros alcanzados hasta el momento, e incluso las dificultades, son valorados como parte del proceso de aprendizaje y crecimiento y por tanto, consideran importante seguir aprendiendo y mejorando cada día.

En Cooptominé como en Asosantuario, reconocen como muy valiosos el apoyo y constancia de Fundesot, así como los contenidos y metodologías de la capacitación. El manejo que tuvo Fundesot en su trabajo con la comunidad, deja claro una vez más, que la mejor manera de transformar una comunidad es la cercanía con ella. Es el hacerse parte del grupo, conociendo sus problemas, viviendo sus dificultades y gozando con sus éxitos, como bien lo plantea la etnografía descrita en la propuesta metodológica.

A pesar de que se evidencian, en general, mejores relaciones con la EEB (Empresa de Energía de Bogotá), sale a flote el inconformismo que hay por su falta de atención a las necesidades de la comunidad, lo cual se ratifica con lo expuesto por algunos de los representantes de las empresas, en cuanto hizo y hace falta un mayor seguimiento del proceso y un mayor compromiso por parte de éstas con las comunidades vecinas.

Otra punto importante para resaltar es el hecho de que según los documentos y entrevistas a los representantes de las empresas, hay claridad frente a que el pastoreo era una de las causas de la afectación al ecosistema, más no la única. Por lo tanto sería importante, conocer qué otras acciones se adelantan para disminuir los impactos y qué compromisos están adquiriendo las otras partes responsables de la contaminación a este cuerpo de agua, como las administraciones que aún no resuelven el vertimiento de aguas servidas, el flujo de residuos químicos por la explotación minera y los cultivos no orgánicos, así como el inadecuado manejo de residuos sólidos que muchas veces vienen a parar al embalse.

Un asunto fundamental, que se hace evidente en las entrevistas y en los talleres de cartografía social, es que el proceso fue positivo para la relación entre la EEB y la comunidad, básicamente porque reconocen que nunca la empresa había tenido en cuenta el trabajo del componente social. Se valora ese acercamiento y los resultados obtenidos, pero se añora una mayor presencia y apoyo por parte de esta empresa vecina, que tanto podría aportar en el crecimiento de una comunidad, y que como 
también mencionan, puede ser un verdadero aliado para el cuidado del embalse. Valdría la pena retomar algunas capacitaciones como lo proponen, y generar un programa social que vincule a la comunidad en los diferentes procesos que ayuden a disminuir las presiones sobre el ecosistema, generando más amigos que enemigos.

Por último, es importante reflexionar sobre la poca valoración del trabajo de los campesinos. Una vez más e igual que en todas las regiones del país, los campesinos productores de leche, en este caso, son los que más se esfuerzan, en las condiciones más difíciles y sin embargo son los que menos ganan en esa cadena productiva. Se hace evidente aquí lo expuesto por Polan Lacky, respecto a la importancia de la educación y la organización, para avanzar en relaciones más equitativas y en el logro de mejores condiciones de vida en el campo.

\section{Conclusiones}

El estudio permitió obtener Conclusiones importantes acerca del papel de la educación ambiental y la organización comunitaria, como herramientas pedagógicas fundamentales para la resolución del conflicto, la disminución del impacto sobre el ecosistema y el aumento de la producción lechera, fuente de ingresos básica de la población.

Se destacan entre estas conclusiones:

- Un conflicto que fue solucionado entre los años 2006 y 2008, muestra después de 5 años a una población que reconoce como superadas las condiciones pecuarias de la comunidad y cómo se modificaron para beneficio de todas las partes.

- Las empresas generaron procesos que permitieron reconocer las prácticas de la comunidad y a la vez motivar y crear alternativas unidas a los intereses para cambiar las prácticas inadecuadas.

- Si en principio, se tenían bajas expectativas y poca credibilidad frente al proceso, poco a poco fueron comprendiendo sus posibilidades, alternativas y visualizando sus posibilidades de cambio, siempre con el acompañamiento de las empresas a través del operador local.

- Los procesos de formación activados por las empresas, devinieron en procesos autónomos de construcción de comunidad, que permiten hablar de una pedagogía que se construye socialmente a partir del diálogo de saberes y del reconocimiento de las prácticas y sus posibilidades de transformación. 
- El estudio hizo evidente la importancia pedagógica de la educación ambiental y la participación comunitaria en la resolución de un conflicto socioambiental, y por lo tanto, en la recuperación de un ecosistema, así como el valor incalculable de la pedagogía en el mejoramiento de la producción, dentro del marco del desarrollo sostenible.

- El proceso mostró el efecto del empoderamiento y del liderazgo por parte de la comunidad en su propio beneficio y la eficacia del trabajo colaborativo y de la construcción colectiva, para el logro de los objetivos propuestos. 


\section{Referencias}

Empresa de Energía de Bogotá. (2006). Plan de Manejo Ambiental del Embalse de Tominé en Operación. Bogotá.

García J. (2012). Lo que es y no es la pedagogía. La columna pedagógica. Un espacio dedicado al análisis y reflexión sobre temas pedagógicos y educativos. Méjico. Recuperado en http://lacolumnapedagogica.blogspot.mx/2012/04/breve-explicacion-de-lo- que-es-y-loque.html, el 12 de septiembre de 2012.

Instituto Alexander Von Humboldt (1998). Hacia la conservación de los Humedales de Colombia. Biosíntesis, (9).

Polan, L. (2006). El verdadero libro de los pobres rurales. Desarrollo agropecuario: de la dependencia al protagonismo del agricultor. Recuperado en www.polanlacki.com.br/agroesp. Consultado el 10 de agosto de 2013.

Polan, L. (2012). Los agricultores y sus nuevas prioridades: exigir una educación útil, profesionalizarse y deponer el individualismo. Recuperado en www.polanlacki. com.br/esp/artigosesp/prioridadesesp.htm.

Ministerio de Ambiente, Vivienda y Desarrollo Territorial y Ministerio de Educación Nacional. (2003). Política Nacional de Educación Ambiental. Bogotá.

Pérez, G. (2004). Pedagogía social y educación social. Construcción científica e intervención práctica. España: Ediciones Narsea, S. A.

Petrus, A. (Ed.). (1997).. Pedagogía Social. Barcelona: Editorial Ariel S.A.

Pujol, R.M. y Muñoz, L.C. (coords.). (2007). Nuevas tendencias en investigaciones en educación ambiental. Madrid: Organismo Autónomo Parques Nacionales. Ministerio de Medio Ambiente, Doctorado universitario en educación ambiental. Naturaleza y Parques Nacionales. Serie educación ambiental.

Quintana, A. El conflicto socio-ambiental y estrategias de manejo. Instituto de estudios para el desarrollo y la paz INDEPAZ. Recuperado en www.fuhem.es/.../Conflictos.../conflicto_ socioambiental_estrategias\%20_...

Recibido: 7 octubre 2012

Aceptado: 10 diciembre 2012

Como citar:

Torres, C. y Torres, C.M. (2013). “La pedagogía

social, una mirada para la resolución de un

conflicto socio-ambiental"

Praxis Pedagógica 14, 71-81 